\section{Prevalência e fatores associados à obesidade abdominal em indivíduos na faixa etária de 25 a 59 anos do Estado de Pernambuco, Brasil}

\author{
Prevalence of abdominal obesity and associated \\ factors among individuals 25 to 59 years of age \\ in Pernambuco State, Brazil
}

Prevalencia y factores asociados a la obesidad abdominal en individuos en una franja de edad de 25 a 59 años del estado de Pernambuco, Brasil
Claudia Porto Sabino Pinho 1

Alcides da Silva Diniz 1

Ilma Kruze Grande de Arruda 1

Malaquias Batista Filho 2

Poliana Cabral Coelho 1

Leopoldina Augusta de Souza Sequeira 1

Pedro Israel Cabral de Lira 1

\footnotetext{
1 Universidade Federal de Pernambuco, Recife, Brasil.

2 Instituto de Medicina Integral Prof. Fernando Figueira, Recife, Brasil.

Correspondência C. P. S. Pinho Universidade Federal de Pernambuco.

Rua Bianor de Oliveira 262 Recife, $P E$

52040-350, Brasil.

claudiasabinopinho@

hotmail.com
}

\begin{abstract}
In order to estimate the prevalence of abdominal obesity and associated factors in Pernambuco State, Brazil, a cross-sectional population-based study was conducted in 2006, including 1,580 adults 25 to 59 years of age. Abdominal obesity was defined as waist circumference $(W C) \geq 80 \mathrm{~cm}$ in women and $\geq 94 \mathrm{~cm}$ in men. The conceptual model included demographic, socioeconomic, reproductive, and behavioral variables. Prevalence of abdominal obesity was $27.1 \%$ (95\% CI: 23.830.7) in males and 69.9\% (95\%CI: 66.8-72.8) in females $(p<0.001)$. Multivariate analysis showed higher prevalence in men 50 years or older in the metropolitan area and those with higher income, former smokers, and drinkers. Among women, obesity was more prevalent above 30 years of age, in former smokers, and in women with first pregnancy before age 18. Central adiposity is clearly a multifactor condition in the State of Pernambuco, and the determinants of obesity differ between the sexes.
\end{abstract}

Abdominal Obesity; Waist Circumference; Epidemiologic Factors; Adult

\section{Resumo}

Com o objetivo de estimar a prevalência de obesidade abdominal e avaliar os fatores associados em adultos do Estado de Pernambuco, Brasil, foi realizado, em 2006, um estudo transversal, de base populacional, envolvendo 1.580 indivíduos na faixa etária de 25-59 anos. A obesidade abdominal foi determinada pela circunferência da cintura $\geq 80 \mathrm{~cm}$ para mulheres $e \geq 94 \mathrm{~cm}$ para homens. O modelo conceitual considerou variáveis socioeconômicas, demográficas, reprodutivas e comportamentais. A prevalência de obesidade abdominal foi de 27,1\% (IC95\%: 23,8-30,7) no sexo masculino e 69,9\% (IC95\%: 66,8-72,8) no feminino $(p<0,001)$. A análise ajustada revelou maior prevalência em homens a partir de 50 anos, da região metropolitana, de maior renda, ex-fumantes e consumidores de bebidas alcoólicas. Entre as mulheres, foi mais prevalente a partir de 30 anos, em ex-fumantes e naquelas com a primeira gestação antes de 18 anos. Fica evidente a gravidade e a multifatorialidade do problema da adiposidade central no Estado de Pernambuco, além de ser possível destacar que os determinantes da obesidade são diferentes entre os sexos.

Obesidade Abdominal; Circunferência da Cintura; Fatores Epidemiológicos; Adulto 


\section{Introdução}

A obesidade é uma doença crônica não transmissível caracterizada pelo excesso de gordura corporal e resultante do desequilíbrio prolongado entre o consumo alimentar e o gasto energético 1,2. Atualmente é um grave problema de saúde pública, atingindo proporções epidêmicas tanto em países desenvolvidos como em nações e regiões em desenvolvimento 2 , com grande impacto sobre o padrão de morbidade de populações adultas 3 .

A obesidade abdominal ou andróide, isto é, o aumento de tecido adiposo na região abdominal, é considerada um fator de risco independente para diversas morbidades, representando risco diferenciado quando comparada com outras formas de distribuição de gordura corporal 4 .

Embora já existam métodos mais sofisticados de avaliação da gordura abdominal, deve-se considerar que do ponto de vista epidemiológico a circunferência da cintura é a medida fácil e de baixo custo para uso em estudos populacionais 4,5. Além disso, comparada com outros indicadores antropométricos é a melhor preditora da gordura visceral localizada na região abdominal, que se mostra fortemente correlacionada à maioria dos fatores de risco metabólico 6 .

No Brasil, estudos de base populacional têm mostrado que fatores sociodemográficos e comportamentais estão associados ao aumento do peso corporal. Entretanto, ainda são escassos os estudos acerca do papel desses determinantes na distribuição da gordura corporal. Além disso, poucos estados brasileiros dispõem de informações oriundas de amostras representativas enfocando o problema da obesidade abdominal, sobretudo que considere a população das diferentes mesorregiões (urbana e rural). Estudo que avaliou a evolução da obesidade geral e abdominal em uma cidade do sul do país entre os anos 2000 e 2010, observou uma estabilidade da prevalência de obesidade abdominal em ambos os sexos no período analisado 7 .

Nesse contexto, este trabalho objetiva estimar a prevalência da obesidade abdominal e os fatores associados na população adulta do Estado de Pernambuco, Brasil, como uma contribuição para caracterizar sua importância e compreender as relações causais que devem ser destacadas como bases estratégicas para o enfrentamento do problema, além de possibilitar que as informações sejam monitoradas e que tendências do comportamento da obesidade abdominal sejam traçadas.

\section{Materiais e métodos}

Foi realizado um estudo de corte transversal, de base populacional, no período de maio a outubro de 2006, envolvendo adultos de ambos os sexos, na faixa etária de 25-59 anos, provenientes de áreas urbana e rural do Estado de Pernambuco. Foram excluídos os indivíduos que apresentaram alguma limitação física que impossibilitasse a aferição das medidas antropométricas, as gestantes e mulheres que tiveram filhos nos seis meses anteriores à coleta.

Para definição do tamanho amostral foi considerada a prevalência de obesidade abdominal encontrada em estudo realizado com adultos de ambos os sexos em uma cidade do Nordeste brasileiro ${ }^{3}$, estimada em $35,7 \%$ das mulheres e $12,9 \%$ dos homens. O cálculo da amostra foi realizado no software Epi Info, versão 6.04 (Centers for Disease Control and Prevention, Atlanta, Estados Unidos), sendo considerada a prevalência de $12,9 \%$ de obesidade abdominal encontrada nos homens nordestinos, um erro de estimação de $3 \%$, um nível de $95 \%$ de confiança, e um efeito do desenho da ordem de 3,0 , perfazendo um total mínimo de 1.439 indivíduos a serem estudados. Para compensar eventuais perdas e para permitir um melhor nível de estratificação das variáveis independentes, esse tamanho amostral foi aumentado em $25 \%$, resultando em uma amostra final de 1.800 pessoas.

O processo de seleção da amostra (probabilística e estratificada) desenvolveu-se em três estágios: (1) sorteio dos municípios; (2) sorteio dos setores censitários (unidades territoriais de amostragem demarcadas pelo IBGE); (3) sorteio aleatório dos domicílios dentro de cada setor censitário para selecionar as famílias, seguindose a incorporação de outras famílias no sentido anti-horário até completar a quota amostral prevista para cada setor (40 \pm 5 famílias).

Com base no sorteio aleatório foram selecionados 18 municípios e considerados três estratos geográficos: Região Metropolitana do Recife, interior urbano e interior rural. Para definição da quantidade de setores censitários a serem estudados, foi considerado o número com representações proporcionais à população dos municípios selecionados, figurando 16 setores censitários da Região Metropolitana do Recife, 17 do interior urbano e 12 do interior rural.

A circunferência da cintura foi aferida em duplicata no ponto médio entre a última costela e a crista ilíaca no momento da expiração, com uma fita métrica inextensível de $200 \mathrm{~cm}$ e variação de $0,1 \mathrm{~cm}$, de acordo com o protocolo estabelecido pela Organização Mundial da Saúde 8. A circunferência da cintura (CC) foi aferida pelo mesmo 
avaliador e repetida quando o erro de aferição entre as duas medidas foi maior que $1 \mathrm{~cm}$. O valor resultante das aferições foi a média entre os dois valores mais próximos. A obesidade abdominal foi determinada quando $C C \geq 80 \mathrm{~cm}$ para mulheres e $\geq 94 \mathrm{~cm}$ para homens ${ }^{8}$.

Para padronização da coleta de dados, os antropometristas foram treinados com conteúdo teórico e prático relativo à técnica de mensuração antropométrica da CC. Nessa ocasião, foram aferidos os erros técnicos de medição intra e interavaliador, respectivamente. Para determinar os erros técnicos de medição intra-avaliador, 20 funcionários do Departamento de Nutrição da Universidade Federal de Pernambuco foram avaliados em dois dias distintos pelo mesmo antropometrista. Para a aferição dos erros técnicos de medição interavaliadores, esses funcionários foram avaliados um de cada vez, durante o mesmo período, pelos antropometristas e por um avaliador padrão, professor da disciplina de avaliação do estado nutricional, com ampla experiência em aferição de medidas antropométricas. De forma geral, o resultado de erros técnicos de medição absoluto intra-avaliador e interavaliador para a CC foi menor que $1 \mathrm{~cm}$, mostrando que os antropometristas selecionados para o trabalho de campo apresentavam condições técnicas para assumir este estudo de base populacional.

O modelo conceitual construído para explicar a obesidade abdominal considerou as seguintes variáveis: demográficas: sexo, idade (categorizada nos intervalos de 25-29 anos, 30-39, 40-49, e 50-59 anos); área geográfica da residência (Região Metropolitana do Recife, interior urbano, interior rural); socioeconômicas: escolaridade, em anos completos de estudos (categorizada nos intervalos: 0-4 anos, 5-8 e $\geq 9$ anos) e renda familiar em salário mínimo per capita (estabelecida em quartis de renda e levando-se em conta o salário mínimo vigente na época: $\mathrm{R} \$ 350,00)$; reprodutivas: paridade (categorizada em $<3$ gestações $\mathrm{e} \geq$ 3 gestações), idade da menarca (categorizada nos intervalos: $\leq 11$ anos, 12-13 e $\geq 14$ anos), idade da primeira gestação (< 18 anos e $\geq 18$ anos) e uso de contraceptivo oral (com a resposta dicotômica sim ou não); e comportamentais: tabagismo, consumo de álcool, atividade física e consumo alimentar.

Quanto ao tabagismo foram consideradas as categorias: fumante (o indivíduo que referiu o hábito de fumar), não fumante (o indivíduo que relatou nunca haver fumado) e ex-fumante (o indivíduo que referiu o hábito de fumar em algum momento da vida, mas que não o praticava na ocasião da aplicação do questionário). Para a variável consumo de álcool, avaliou-se o consumo de bebidas alcoólicas nos 30 dias anteriores à aplicação do questionário, sendo considerada a resposta dicotômica sim ou não.

Para determinação do nível de atividade física da população foi utilizado o International Physical Activity Questionnaire (IPAQ - http:// www.ipaq.ki.se/ipaq.htm, acessado em 10/Set/ 2010), 2001, em sua versão curta, que leva em consideração as quatro dimensões da atividade física: no lazer, atividades domésticas, atividades ocupacionais e atividades relacionadas ao deslocamento. Esse instrumento mede a frequência e duração das atividades físicas moderadas, vigorosas e caminhadas realizadas na última semana por pelo menos dez minutos contínuos, incluindo exercícios, esportes, atividades físicas ocupacionais e recreacionais realizadas em casa, no tempo livre, como meio de transporte e no lazer.

Foi construído um escore de atividade física em minutos por semana, somando-se os minutos despendidos em caminhada e atividades de intensidade moderada com os minutos gastos em atividades de intensidade vigorosa multiplicados por dois. Essa estratégia visa a considerar as diferentes intensidades de cada atividade e está de acordo com as recomendações atuais quanto à prática de atividade física. Um escore abaixo de 150 minutos por semana foi o ponto de corte utilizado para classificar os indivíduos como insuficientemente ativos ou sedentários 10.

As informações referentes ao consumo alimentar foram coletadas utilizando-se um questionário de frequência alimentar, e a avaliação do consumo foi realizada com base na metodologia proposta por Fornés et al. 10, na qual o cômputo geral da frequência de consumo é convertido em escores. Foram constituídos três grupos de alimentos: o grupo 1 foi composto por alimentosfonte de fibras, como leguminosas, frutas, legumes e hortaliças, considerados protetores para as doenças cardiovasculares e para o ganho excessivo de peso; o grupo 2 reuniu alimentos-fonte de carboidratos simples (bolo, biscoito, açúcar e refrigerantes); e no grupo 3 foram inseridos os alimentos-fonte de gorduras saturadas (carnes com gordura, frango com pele, vísceras, embutidos, laticínios, gorduras e frituras). Os grupos $2 \mathrm{e}$ 3 foram compostos por alimentos considerados de risco para o desenvolvimento de doenças cardiovasculares e para o ganho de peso excessivo. Os escores de consumo de cada grupo foram categorizados em quartis.

Foram adotados procedimentos para assegurar a qualidade da informação coletada: treinamento teórico e prático, padronização de medidas antropométricas, seleção criteriosa dos entrevistadores e antropometristas e estudo piloto com 80 famílias em 2 setores censitários 
(não incluídos na amostra final). Os dados coletados eram revisados e codificados, visando a detectar falhas no preenchimento, identificar formulários não preenchidos por ausência de algum membro da família ou por outro motivo que exigisse retorno imediato ao domicílio. Ao final do dia, os questionários eram revisados pelo supervisor (segunda revisão) para detecção de falhas de preenchimento, ausência de dados antropométricos e, em seguida, eram repassados ao coordenador do trabalho de campo que realizava uma terceira revisão, para complementar os dados, caso necessário.

O protocolo do estudo foi conduzido respeitando-se os padrões éticos, sendo aprovado pelo Comitê de Ética em Pesquisa em Seres Humanos do Instituto de Medicina Integral Prof. Fernando Figueira (IMIP), sob o no 709/2006. As pessoas que concordaram em participar da pesquisa assinaram o termo de consentimento livre e esclarecido.

A base de dados foi compilada no Programa Epi Info versão 6.04, com dupla entrada e posterior uso do módulo validate para checar eventuais erros de digitação. Para as análises estatísticas, foi empregado o SPSS versão 12.0 (SPSS Inc., Chicago, Estados Unidos).

A análise estatística foi feita em três etapas. Inicialmente, realizou-se uma análise descritiva para caracterizar a distribuição da ocorrência dos eventos, incluindo a frequência de cada variável do estudo. Na segunda etapa, procedeu-se uma análise univariada entre a variável dependente (obesidade abdominal) e as variáveis independentes para determinação da razão de prevalência (RP) e seu respectivo intervalo de $95 \%$ de confiança (IC95\%). Numa terceira etapa, foi realizada a análise multivariada utilizando-se o software Stata/SE 9.0 (Stata Corp., College Station, Estados Unidos), pelo método de regressão de Poisson em que foram incluídas no modelo múltiplo todas as variáveis que se mostraram associadas ao evento de interesse com significância estatística de até $20 \%$. Para aceitação das associações investigadas no modelo final, após os ajustes foi adotado o valor de $\mathrm{p}<0,05$.

\section{Resultados}

Avaliou-se 1.800 indivíduos, dos quais 2,2\% foram excluídos (gestantes, puérperas e incapacidade física) e $10 \%$ foram perdas (ausência de resposta e inconsistência de informações), totalizando 1.580 adultos efetivamente estudados. A mediana da idade foi 33 anos (intervalo interquartil: 29-42), predominando o sexo feminino (58\%) e a baixa escolaridade $(54,3 \%$ com menos de 5 anos completos de estudos). A mediana da renda familiar per capita (em salários mínimos - SM) foi de 0,25 (intervalo interquartil $=0,12-0,45$ ). Em relação às características comportamentais foi observada uma prevalência de $22,3 \%$ de fumantes, $15,1 \%$ de ex-fumantes e $28,5 \%$ de indivíduos insuficientemente ativos.

A prevalência de obesidade abdominal nos adultos do Estado de Pernambuco foi de 51,9\% (IC95\%: 49,4-54,4), sendo maior no sexo feminino $(\mathrm{p}<0,001)$ (Tabela 1$)$.

A análise univariada para o sexo masculino evidenciou maior prevalência de obesidade abdominal a partir da quarta década de vida, em ex-fumantes, naqueles insuficientemente ativos, provenientes da Região Metropolitana do Recife e na maior faixa de escolaridade e renda (Tabela 2).

Em relação ao sexo feminino, na análise bruta, foi verificada maior prevalência da obesidade abdominal a partir da terceira década de vida, na menor faixa de escolaridade e não foi observada associação com a renda. Verificouse ainda maior prevalência da obesidade abdominal em mulheres ex-fumantes, naquelas com

Tabela 1

Prevalência de obesidade abdominal em adultos na faixa etária de 25-59 anos. Pernambuco, Brasil, 2006.

\begin{tabular}{|c|c|c|c|c|c|c|c|c|c|}
\hline \multirow[t]{2}{*}{ Indicador antropométrico } & \multicolumn{3}{|c|}{ Total } & \multicolumn{3}{|c|}{ Homens } & \multicolumn{3}{|c|}{ Mulheres } \\
\hline & $\mathrm{n}$ & $\%$ & IC95\% & $n$ & $\%$ & IC95\% & $\mathrm{n}$ & $\%$ & IC95\% \\
\hline Circunferência da cintura $(\mathrm{cm})$ & 1.580 & & & 664 & & & 916 & & \\
\hline Normal & 760 & 48,1 & $46,2-51,2$ & 484 & 72,9 & $69,3-76,2$ & 276 & 30,1 & $27,2-33,2$ \\
\hline Elevada * & 820 & 51,9 & $49,4-54,4$ & 180 & 27,1 & $23,8-30,7$ & 640 & 69,9 & $66,8-72,8$ \\
\hline
\end{tabular}

IC95\%: intervalo de 95\% de confiança.

* Homens $\geq 94 \mathrm{~cm}$ e Mulheres $\geq 80 \mathrm{~cm}$.

Teste qui-quadrado de Pearson: $p<0,001$. 
Razão de prevalência (RP) da obesidade abdominal * em homens na faixa etária de 25-59 anos, de acordo com as características demográficas, socioeconômicas e comportamentais. Pernambuco, Brasil, 2006.

\begin{tabular}{|c|c|c|c|c|c|c|c|}
\hline \multirow[t]{2}{*}{ Variáveis } & \multicolumn{2}{|c|}{ Normal } & \multicolumn{2}{|c|}{ Elevada * } & \multirow[t]{2}{*}{$\mathrm{RP}$} & \multirow[t]{2}{*}{ IC95\% } & \multirow[t]{2}{*}{ Valor de $p$} \\
\hline & $\mathrm{n}$ & $\%$ & $\mathrm{n}$ & $\%$ & & & \\
\hline Idade (anos) & & & & & & & 0,072 ** \\
\hline $25-29$ & 132 & 76,3 & 41 & 23,7 & 1,00 & - & \\
\hline $30-39$ & 210 & 72,9 & 78 & 27,1 & 1,14 & $0,99-1,27$ & \\
\hline $40-49$ & 90 & 74,4 & 31 & 25,6 & 1,08 & $1,11-1,47$ & \\
\hline $50-59$ & 52 & 63,4 & 30 & 36,6 & 1,54 & $1,23-1,65$ & \\
\hline Área de residência & & & & & & & $<0,001$ ** \\
\hline Região Metropolitana do Recife & 103 & 63,2 & 60 & 36,8 & 1,00 & - & \\
\hline Interior urbano & 89 & 58,6 & 63 & 41,4 & 1,13 & $0,85-1,48$ & \\
\hline Interior rural & 292 & 83,7 & 57 & 16,3 & 0,44 & $0,33-0,61$ & \\
\hline \multicolumn{8}{|l|}{ Escolaridade (anos de estudo) } \\
\hline $0-4$ & 302 & 80,5 & 73 & 19,5 & 0,48 & $0,82-1,59$ & $<0,001 \neq$ \\
\hline $5-8$ & 84 & 66,7 & 42 & 33,3 & 0,83 & $0,72-1,62$ & \\
\hline$\geq 9$ & 89 & 59,7 & 60 & 40,3 & 1,00 & - & \\
\hline \multicolumn{8}{|l|}{ Renda familiar per capita (salários mínimos) } \\
\hline$\leq 0,12$ & 155 & 87,1 & 23 & 12,9 & 1,00 & - & $<0,001 * *$ \\
\hline $0,12-0,25$ & 127 & 74,3 & 44 & 25,7 & 1,99 & $1,26-3,15$ & \\
\hline $0,25-0,45$ & 110 & 74,8 & 37 & 25,2 & 1,95 & $1,21-3,12$ & \\
\hline$\geq 0,45$ & 86 & 53,4 & 75 & 46,6 & 3,61 & $2,38-5,46$ & \\
\hline Tabagismo & & & & & & & 0,013 ** \\
\hline Fumante & 145 & 78,8 & 39 & 21,2 & 1,00 & - & \\
\hline Não fumante & 252 & 72,2 & 97 & 27,8 & 1,31 & $0,95-1,82$ & \\
\hline Ex-fumante & 87 & 66,4 & 44 & 33,6 & 1,58 & $1,10-2,29$ & \\
\hline Consumo de álcool nos últimos 30 dias & & & & & & & 0,092 *** \\
\hline Não & 209 & 70,8 & 86 & 29,2 & 1,00 & - & \\
\hline Sim & 236 & 76,9 & 71 & 23,1 & 0,79 & $0,61-1,04$ & \\
\hline Atividade física \# & & & & & & & $0,012 * \star \star$ \\
\hline Suficientemente ativo & 379 & 75,3 & 124 & 24,7 & 1,00 & - & \\
\hline Insuficientemente ativo & 105 & 65,2 & 56 & 34,8 & 1,41 & $1,09-1,83$ & \\
\hline Consumo alimentar (escore) \# & & & & & & & 0,330 ** \\
\hline \multicolumn{8}{|l|}{ Grupo 1 (quartis) } \\
\hline$\leq 1,67$ & 123 & 76,9 & 37 & 23,1 & 0,80 & $0,55-1,15$ & \\
\hline $1,67-2,33$ & 112 & 70,4 & 47 & 29,6 & 1,02 & $0,73-1,42$ & \\
\hline $2,33-3,17$ & 125 & 73,1 & 46 & 26,9 & 0,93 & $0,66-1,30$ & \\
\hline$\geq 3,17$ & 122 & 70,9 & 50 & 29,1 & 1,00 & - & \\
\hline Grupo 2 (quartis) & & & & & & & 0,840 ** \\
\hline$\leq 3,03$ & 121 & 71,6 & 48 & 28,4 & 1,00 & - & \\
\hline $3,03-4,20$ & 119 & 73,5 & 43 & 26,5 & 0,93 & $0,65-1,32$ & \\
\hline $4,20-5,33$ & 115 & 73,7 & 41 & 26,3 & 0,93 & $0,69-1,36$ & \\
\hline$\geq 5,33$ & 127 & 72,6 & 48 & 27,4 & 0,97 & $0,66-1,33$ & \\
\hline Grupo 3 (quartis) & & & & & & & 0,009 ** \\
\hline$\leq 2,47$ & 134 & 77,9 & 38 & 22,1 & 1,00 & - & \\
\hline $2,47-3,50$ & 126 & 76,8 & 38 & 23,2 & 1,05 & $0,71-1,56$ & \\
\hline $3,50-4,53$ & 109 & 68,6 & 50 & 31,4 & 1,42 & $0,99-2,05$ & \\
\hline$\geq 4,53$ & 109 & 67,3 & 53 & 32,7 & 1,48 & $1,04-2,12$ & \\
\hline
\end{tabular}

IC95\%: intervalo de $95 \%$ de confiança.

$* \geq 94 \mathrm{~cm}$;

** Teste qui-quadrado de tendência linear;

*** Teste qui-quadrado de Pearson;

\# Suficientemente ativos: caminhada ou atividades moderadas ou vigorosas por tempo $\geq 150$ minutos/semana. Insuficientemente ativos: sedentários e indivíduos com atividades moderada, vigorosa ou caminhada < 150 minutos/semana; Grupo 1: frutas, legumes, hortaliças e leguminosas. Grupo 2: bolo, biscoito, açúcar e refrigerante. Grupo 3: carnes com gordura, frango com pele, vísceras, embutidos, leite e derivados, gorduras e frituras. 
a primeira gestação antes dos 18 anos, e entre aquelas com menor consumo de alimentos do Grupo 2 (alimentos-fonte de carboidratos simples) (Tabela 3).

Na análise multivariada para o sexo masculino mantiveram associação com a obesidade abdominal as variáveis: idade, estrato geográfico da residência, renda, tabagismo e consumo de álcool. Para o sexo feminino, a análise ajustada evidenciou associação com a idade, o tabagismo e a idade da primeira gestação (Tabela 4).

\section{Discussão}

A obesidade, sobretudo a abdominal, predispõe a pessoa a uma série de fatores de risco cardiovasculares por associar-se com grande frequência a condições como dislipidemias, hipertensão arterial, resistência à insulina e diabetes mellitus que favorecem a ocorrência de eventos cardiovasculares, particularmente os coronarianos ${ }^{6}$.

A alta prevalência de indivíduos com obesidade abdominal revelada nesta investigação reflete a magnitude do problema, que tem atingido contornos epidêmicos tanto em países em desenvolvimento como entre os desenvolvidos. No Nordeste, estudos representativos da população adulta de Salvador (Bahia) 3 e do Estado de Maranhão 11 registraram prevalências inferiores ( $28,1 \%$ e $46,3 \%$, respectivamente), demonstrando que o problema pode ter magnitude potencialmente mais elevada em algumas populações, e que tem gravidade importante no Estado de Pernambuco. Comparando-se ao problema do excesso de peso, estudo recente 12 destacou, entre os pernambucanos, prevalência semelhante $(51,1 \%)$ à descrita neste estudo $(51,9 \%)$.

A obesidade abdominal, claramente, tem maior predominância em mulheres em todo o

Tabela 3

Razão de prevalência (RP) da obesidade abdominal * em mulheres na faixa etária de 25-59 anos, de acordo com as características demográficas, socioeconômicas, reprodutivas e comportamentais. Pernambuco, Brasil, 2006.

\begin{tabular}{|c|c|c|c|c|c|c|c|}
\hline \multirow[t]{2}{*}{ Variáveis } & \multicolumn{2}{|c|}{ Normal } & \multicolumn{2}{|c|}{ Elevada * } & \multirow[t]{2}{*}{ RP } & \multirow[t]{2}{*}{ IC95\% } & \multirow[t]{2}{*}{ Valor de $p$} \\
\hline & $\mathrm{n}$ & $\%$ & $\mathrm{n}$ & $\%$ & & & \\
\hline Idade (anos) & & & & & & & $<0,001 * *$ \\
\hline $25-29$ & 133 & 42,6 & 179 & 57,4 & 1,00 & - & \\
\hline $30-39$ & 102 & 29,6 & 243 & 70,4 & 1,23 & $1,09-1,38$ & \\
\hline $40-49$ & 32 & 18,9 & 137 & 81,1 & 1,41 & $1,25-1,59$ & \\
\hline $50-59$ & 9 & 10,0 & 81 & 90,0 & 1,57 & $1,39-1,76$ & \\
\hline Área de residência & & & & & & & 0,578 ** \\
\hline Região Metropolitana do Recife & 79 & 30,5 & 180 & 69,5 & 1,00 & - & \\
\hline Interior urbano & 76 & 32,1 & 161 & 67,6 & 0,98 & $0,87-1,10$ & \\
\hline Interior rural & 121 & 28,8 & 299 & 71,2 & 1,02 & $0,93-1,11$ & \\
\hline Escolaridade (anos de estudo) & & & & & & & $0,001 * *$ \\
\hline $0-4$ & 127 & 26,8 & 347 & 73,2 & 1,23 & $1,08-1,39$ & \\
\hline $5-8$ & 61 & 27,4 & 162 & 72,6 & 1,22 & $1,06-1,39$ & \\
\hline$\geq 9$ & 87 & 40,3 & 129 & 59,7 & 1,00 & - & \\
\hline Renda familiar per capita (salários mínimos) & & & & & & & $0,164 * *$ \\
\hline$\leq 0,12$ & 70 & 32,6 & 145 & 67,4 & 1,00 & - & \\
\hline $0,12-0,25$ & 74 & 30,7 & 167 & 69,3 & 1,03 & $0,91-1,16$ & \\
\hline $0,25-0,45$ & 69 & 31,4 & 151 & 68,6 & 1,02 & $0,89-1,16$ & \\
\hline$\geq 0,45$ & 59 & 26,0 & 168 & 74,0 & 1,10 & $0,97-1,24$ & \\
\hline Tabagismo & & & & & & & 0,006 ** \\
\hline Fumante & 55 & 32,5 & 114 & 67,5 & 1,00 & - & \\
\hline Não fumante & 205 & 32,1 & 433 & 67,9 & 1,01 & $0,89-1,13$ & \\
\hline Ex-fumante & 16 & 14,8 & 92 & 85,2 & 1,26 & $1,11-1,44$ & \\
\hline Consumo de álcool nos últimos 30 dias & & & & & & & $0,642 * \star \star$ \\
\hline Não & 52 & 31,7 & 112 & 68,3 & 1,00 & - & \\
\hline Sim & 212 & 29,9 & 498 & 70,1 & 1,03 & $0,92-1,15$ & \\
\hline
\end{tabular}

(continua) 


\begin{tabular}{|c|c|c|c|c|c|c|c|}
\hline \multirow[t]{2}{*}{ Variáveis } & \multicolumn{2}{|c|}{ Normal } & \multicolumn{2}{|c|}{ Elevada * } & \multirow[t]{2}{*}{$\mathrm{RP}$} & \multirow[t]{2}{*}{ IC95\% } & \multirow[t]{2}{*}{ Valor de $p$} \\
\hline & $\mathrm{n}$ & $\%$ & $\mathrm{n}$ & $\%$ & & & \\
\hline 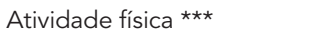 & & & & & & & $0,840 * \star \star$ \\
\hline Suficientemente ativo & 171 & 30,4 & 392 & 69,6 & 1,00 & - & \\
\hline Insuficientemente ativo & 105 & 29,7 & 248 & 70,3 & 1,01 & $0,92-1,10$ & \\
\hline \multicolumn{8}{|l|}{ Consumo alimentar (escore) \# } \\
\hline Grupo 1 (quartis) & & & & & & & $0,887 * \star$ \\
\hline$\leq 1,67$ & 73 & 31,1 & 162 & 69,9 & 1,00 & $0,88-1,13$ & \\
\hline $1,67-2,33$ & 59 & 28 & 152 & 72 & 1,04 & $0,92-1,18$ & \\
\hline $2,33-3,17$ & 76 & 30,6 & 172 & 69,4 & 1,00 & $0,89-1,13$ & \\
\hline$\geq 3,17$ & 68 & 30,9 & 152 & 69,1 & 1,00 & - & \\
\hline Grupo 2 (quartis) & & & & & & & 0,042 ** \\
\hline$\leq 3,03$ & 51 & 23,6 & 165 & 76,4 & 1,00 & - & \\
\hline $3,03-4,20$ & 74 & 29,5 & 177 & 70,5 & 0,92 & $0,83-1,03$ & \\
\hline $4,20-5,33$ & 79 & 35,0 & 147 & 65,0 & 0,85 & $0,75-0,96$ & \\
\hline$\geq 5,33$ & 72 & 32,4 & 150 & 67,6 & 0,88 & $0,79-0,99$ & \\
\hline Grupo 3 (quartis) & & & & & & & $0,040 * \star$ \\
\hline$\leq 2,47$ & 59 & 25,8 & 170 & 74,2 & 1,00 & $0,87-1,08$ & \\
\hline $2,47-3,50$ & 64 & 28,1 & 164 & 71,9 & 0,97 & $0,80-1,01$ & \\
\hline $3,50-4,53$ & 74 & 33,2 & 149 & 66,8 & 0,90 & $0,80-1,01$ & \\
\hline$\geq 4,53$ & 78 & 33,3 & 156 & 66,7 & 0,90 & - & \\
\hline Paridade & & & & & & & 0,219 *** \\
\hline$<3$ gestações & 156 & 37,6 & 259 & 62,4 & 1,00 & - & \\
\hline$\geq 3$ gestações & 165 & 33,7 & 325 & 66,3 & 1,06 & $0,96-1,17$ & \\
\hline Idade da menarca (anos) & & & & & & & 0,368 ** \\
\hline$\leq 11$ & 43 & 36,1 & 76 & 63,9 & 1,00 & - & \\
\hline $12-13$ & 140 & 37,7 & 231 & 62,3 & 0,97 & $0,83-1,14$ & \\
\hline$\geq 14$ & 122 & 40,4 & 180 & 59,6 & 0,93 & $0,79-1,10$ & \\
\hline Idade da 1a gestação (anos) & & & & & & & 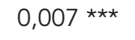 \\
\hline$<18$ & 64 & 30,6 & 145 & 69,4 & 1,00 & - & \\
\hline$\geq 18$ & 241 & 41,1 & 345 & 58,9 & 0,85 & $0,76-0,95$ & \\
\hline Uso de contraceptivo oral & & & & & & & $0,365 * \star \star$ \\
\hline Não & 223 & 37,6 & 370 & 62,4 & 1,00 & - & \\
\hline Sim & 88 & 41,1 & 126 & 58,9 & 0,94 & $0,83-1,17$ & \\
\hline
\end{tabular}

IC95\%: intervalo de $95 \%$ de confiança.

$\star \geq 80 \mathrm{~cm}$;

** Teste qui-quadrado de tendência linear;

*** Teste qui-quadrado de Pearson:

\# Suficientemente ativos: caminhada ou atividades moderadas ou vigorosas por tempo $\geq 150$ minutos/semana. Insuficientemente ativos: sedentários e indivíduos com atividades moderada, vigorosa ou caminhada < 150 minutos/semana; Grupo 1: frutas, legumes, hortaliças e leguminosas. Grupo 2: bolo, biscoito, açúcar e refrigerante. Grupo 3: carnes com gordura, frango com pele, vísceras, embutidos, leite e derivados, gorduras e frituras.

mundo. Essa heterogeneidade na distribuição da obesidade abdominal entre os sexos é consistente com os resultados descritos por outros autores 11,13, e a maior prevalência em mulheres poderia ser atribuída à maior concentração de gordura corporal comumente relatada no sexo feminino, gestações, diferenças hormonais e ao climatério 14 . Além disso, com o processo de envelhecimento e redistribuição progressiva da gordura, as mulheres passam a acumular mais gordura subcutânea, embora tendam a perdêla mais tardiamente com a progressão da idade quando comparadas aos homens 15.

A associação da obesidade abdominal com a idade poderia ser explicada, em parte, por fatores como o declínio natural do hormônio do crescimento, da taxa metabólica basal e da redução natural do nível de atividade física ${ }^{14}$. Adicio- 
Modelo final (regressão de Poisson) dos fatores associados à obesidade abdominal * em mulheres e homens na faixa etária de 25-59 anos. Pernambuco, Brasil, 2006.

\begin{tabular}{|c|c|c|c|}
\hline Variáveis & RP & IC95\% & Valor de $p$ \\
\hline \multicolumn{4}{|l|}{ Homens } \\
\hline Idade (anos) & & & 0,045 \\
\hline $25 \mid-30$ & 1,00 & - & \\
\hline $30 \mid-40$ & 1,24 & $0,88-1,74$ & \\
\hline $40 \mid-50$ & 1,08 & $0,71-1,66$ & \\
\hline $50 \mid-59$ & 1,75 & $1,16-2,66$ & \\
\hline \multicolumn{4}{|l|}{ Área de residência } \\
\hline Região Metropolitana do Recife & 1,00 & - & $<0,001$ \\
\hline Interior urbano & 1,32 & $0,96-1,80$ & \\
\hline Interior rural & 0,64 & $0,43-0,94$ & \\
\hline Renda familiar per capita (quartis) & & & 0,008 \\
\hline$\leq 0,12$ & 1,00 & - & \\
\hline $0,12-0,25$ & 1,50 & $0,92-2,46$ & \\
\hline $0,25-0,45$ & 1,53 & $0,93-2,54$ & \\
\hline$\geq 0,45$ & 2,47 & $1,52-4,01$ & \\
\hline Tabagismo & & & 0,038 \\
\hline Fumante & 1,00 & - & \\
\hline Não fumante & 1,35 & $0,97-1,90$ & \\
\hline Ex-fumante & 1,67 & $1,12-2,48$ & \\
\hline \multicolumn{4}{|l|}{ Consumo de álcool nos últimos 30 dias } \\
\hline Não & 1,00 & - & 0,043 \\
\hline Sim & 1,32 & $1,01-1,73$ & \\
\hline \multicolumn{4}{|l|}{ Mulheres } \\
\hline Idade (anos) & & & $<0,001$ \\
\hline $25 \mid-30$ & 1,00 & - & \\
\hline $30 \mid-40$ & 1,22 & $1,08-1,37$ & \\
\hline $40 \mid-50$ & 1,42 & $1,26-1,62$ & \\
\hline $50 \mid-59 * \star$ & - & - & \\
\hline Idade da 1ạ gestação (anos) & & & 0,008 \\
\hline$<18$ & 1,00 & - & \\
\hline$\geq 18$ & 0,87 & $0,79-0,96$ & \\
\hline Tabagismo & & & 0,028 \\
\hline Fumante & 1,00 & - & \\
\hline Não fumante & 1,06 & $0,93-1,22$ & \\
\hline Ex-fumante & 1,21 & $1,04-1,41$ & \\
\hline
\end{tabular}

IC95\%: intervalo de 95\% de confiança; RP: razão de prevalência.

* Circunferência de cintura $\geq 80 \mathrm{~cm}$ para mulheres e circunferência de cintura $\geq 94 \mathrm{~cm}$ para homens;

** Esta categoria de idade não entrou no modelo por problema de colinearidade com a variável depende.

nalmente, sabe-se que há uma redistribuição de gordura corporal com o avançar da idade, levando a um maior acúmulo de gordura visceral e intra-abdominal, enquanto a gordura subcutânea tende a diminuir nos membros 15 . Essa tendência linear de aumento da prevalência da obesidade abdominal com o avanço das faixas de idade em ambos os sexos também foi descrita por outros autores 4,14, e é um fenômeno observado tanto quando a obesidade é aferida por meio do IMC como quando mensurada por indicadores de obesidade central em adultos 4 .

Vários são os estudos demonstrando que os determinantes da obesidade são diferentes entre os sexos 16,17. As principais diferenças dizem respeito à influência das condições socioeconô- 
micas 18 . Há uma tendência de que homens de maior renda e escolaridade apresentem maiores prevalências de obesidade, enquanto que as mulheres de maior nível socioeconômico apresentariam menores prevalências 11,12. Neste estudo, essas diferenças foram detectadas: aumento da prevalência da obesidade abdominal pari passu com a progressão da renda e escolaridade entre os homens; e no sexo feminino menores prevalências do problema foram reveladas nas mulheres com mais anos de estudos. Portanto, o maior nível educacional, que possibilita maior conhecimento e acesso a um estilo de vida saudável, proporcionaria apenas ao sexo feminino estabilidade do problema do acúmulo de gordura na região abdominal. É possível que no Estado de Pernambuco essa tendência comece a se manifestar como resultado das rápidas mudanças que caracterizam o processo de transição nutricional no Brasil e no Nordeste.

A menor prevalência da obesidade abdominal encontrada nos homens da região rural do Estado de Pernambuco possivelmente está relacionada a fatores alimentares e ao nível de atividade física. Considerando tratar-se de área rural, pressupõe-se um maior gasto energético, representado pelo tipo de trabalho desenvolvido e pelos deslocamentos percorridos, além de um menor acesso a alimentos industrializados, que comumente têm elevada densidade calórica. Essa combinação beneficiaria o homem rural no processo de acúmulo de gordura abdominal 12 .

São crescentes os estudos que avaliam o impacto das variáveis reprodutivas na determinação da obesidade abdominal. A paridade tem sido constantemente identificada como fator de risco 3,14,19, embora não tenha sido identificada associação neste estudo. Por outro lado, fatores obstétricos como a idade de menarca e a idade ao primeiro parto não têm sido consistentemente investigados. A associação com a idade prematura ao primeiro parto revelada neste trabalho, poderia ser atribuída à ativação precoce dos hormônios relacionados ao ciclo reprodutivo, propiciando assim um aumento da concentração de gordura corporal 20 . Oliveira et al. 19 , ao analisarem os fatores associados à obesidade abdominal em mulheres usuárias de um Centro de Saúde de Belo Horizonte, Minas Gerais, não verificaram associação.

A maior prevalência da obesidade abdominal em ex-fumantes é um resultado que corrobora com os dados de outros estudos 3,14 que demonstram que os fumantes que abandonaram o hábito de fumar são os que apresentam as maiores prevalências do problema. Evidências demonstram que a cessação do tabagismo resulta em aumento de peso corporal tanto em homens como em mulheres, e mais de $75 \%$ dos fumantes ganham peso ao tornarem-se abstinentes 22. É possível que o problema do aumento da gordura corporal após a cessação do tabagismo tenha repercussões além dos aspectos individuais. O eventual impacto em saúde pública começa a ser avaliado como causa que contribui para a epidemia de obesidade em alguns países. Nos Estados Unidos, calcula-se que a fração de obesidade atribuível à cessação do tabagismo esteja na ordem de 6 e 3\% para homens e mulheres, respectivamente 22 .

Apesar de o detalhamento dos mecanismos anteriormente descritos não estar totalmente claro, a maioria dos estudos mostra que a cessação do tabagismo parece resultar em ganho ponderal principalmente pelo aumento da ingestão calórica, embora possa ocorrer ganho ponderal sem aumento de calorias ingeridas 23 . Nessa situação de cessação do tabagismo, ocorre uma diminuição da taxa metabólica basal, podendo variar entre $4 \%$ e $16 \%$, o que representaria menos de $40 \%$ do ganho ponderal 23 . Na fase de cessação, há o consumo preferencial de alimentos especialmente ricos em gorduras e açúcares 24 .

É importante destacar a redução do número de fumantes no Brasil. Este trabalho identificou uma prevalência de tabagismo de $22,3 \%$, e dados dos anos de 1989 e 2003 apontam uma redução nesta prevalência em adultos $\geq 18$ anos de $34,8 \%$ para $22,4 \%$, o que representa uma queda anual relativa de $2,5 \%$. Essa importante redução foi resultado de ações de prevenção e controle do tabagismo, que incluíram medidas educativas, preventivas e regulatórias, como a aprovação, no Congresso Nacional, da Lei no 9294/96, que proíbe o uso de cigarros em recintos de uso coletivo e, a partir de 2000, proibiu a publicidade, a promoção e o patrocínio de produtos do tabaco, restringindo-os aos pontos de venda 25 . Portanto, com o aumento do número de ex-fumantes maior atenção deve ser dada para esta população, que constitui um grupo de risco para o ganho excessivo de peso e acúmulo de gordura abdominal.

O consumo do álcool associou-se à obesidade abdominal apenas para o sexo masculino. Diversos estudos têm avaliado o papel do consumo de bebidas alcoólicas na alteração dos indicadores de obesidade global e abdominal 3,11. Entretanto, ainda não está claro o papel do álcool no mecanismo de determinação da adiposidade, sobretudo pela grande variação metodológica encontrada na literatura relativa à análise da frequência e quantidade do etanol consumido.

A maior prevalência da obesidade abdominal em homens considerados sedentários ou insuficientemente ativos é um resultado esperado, tendo em vista que evidências epidemiológicas indicam relação inversa entre a gordura corporal 
e o gasto energético "não-basal”, que é representado principalmente pela atividade física 19,26. Além disso, é descrito que a gordura corporal é mais favoravelmente distribuída nos indivíduos fisicamente ativos 26 .

O delineamento transversal deste trabalho constitui uma limitação na análise das relações de causa e efeito entre as variáveis de exposição (notadamente as variáveis comportamentais como o nível de atividade física e os hábitos alimentares) e o desfecho (obesidade abdominal), podendo ocasionar possíveis causalidades reversas entre as associações. É provável que a maior prevalência de obesidade abdominal observada entre as mulheres com menor consumo de alimentos-fonte de carboidratos simples seja interpretada sob esse víeis. Nesse caso, uma vez que diagnosticada a obesidade abdominal, a pessoa teria iniciado mudanças no padrão alimentar para o controle e redução da gordura corporal.

Outra provável limitação deste estudo referese ao fato de nossa casuística ter incluído indivíduos adultos apenas a partir dos 25 anos. Tal situação poderia comprometer a representatividade da população adulta em seu conjunto. No entanto, constitui um fato conjuntural justificado pela circunstância de que os dados primários deste estudo foram provenientes de pesquisa desenhada para avaliação de doenças crônicas, optando-se, em virtude da limitação de recursos, pela não inclusão dos indivíduos mais jovens (20-24 anos).

Um aspecto importante a ser discutido refere-se ao ponto de corte adotado para determinar a obesidade abdominal $(\geq 80 \mathrm{~cm}$ para mulheres e $\geq 94 \mathrm{~cm}$ para homens ${ }^{8}$ ), também utilizado por outros autores 3,11 . Optou-se por um ponto de corte de maior sensibilidade, tendo em vista que a obesidade abdominal mesmo em estágios de menor gravidade já reflete um risco aumentado para as doenças cardiovasculares. Além disso, estudo que avaliou a associação entre a CC e a hipertensão arterial sistêmica mostrou que esse ponto de corte apresentou melhor capacidade preditiva do risco de hipertensão arterial sistêmi- ca (HAS) 1, ao passo que o nível 2 de determinação da obesidade abdominal ( $\geq 88 \mathrm{~cm}$ para mulheres e $\geq 102 \mathrm{~cm}$ para homens ${ }^{8}$ ) apresentou baixa sensibilidade para detectar a HAS em ambos os sexos. Portanto, considerando que pequenos aumentos na adiposidade abdominal elevam o risco de doenças cardiovasculares, a utilização de pontos de corte mais sensíveis é importante para a prevenção e controle do problema em termos de saúde pública, evitando que a identificação e adoção de ações ocorram apenas em situação de maior gravidade. Logicamente, a utilização desse ponto de corte mais sensível tem repercussão sobre a prevalência do desfecho estudado. Se o nível 2 de CC fosse considerado como ponto de corte neste trabalho, a prevalência de obesidade abdominal seria reduzida para $32,5 \%$ (11,7\% para homens e 47,5\% para mulheres).

Direcionado à descrição e análise da obesidade abdominal, o estudo delineia, no Nordeste, uma importante caracterização do problema da adiposidade central e seus fatores de riscos, em nível populacional. Oferece, assim, subsídios para aprofundar novas contribuições à pesquisa e, sobretudo, suporte empírico e conceitual para medidas na esfera da saúde pública e do comportamento individual e coletivo. Algumas conclusões estão bem caracterizadas: o risco aumentado da obesidade abdominal em indivíduos de maior idade e em ex-fumantes, em homens de maior nível socioeconômico, da região metropolitana, que consomem bebidas alcoólicas e sedentários, em mulheres de menor escolaridade e com a primeira gestação antes dos 18 anos. Seria esse, portanto, o perfil básico do público-alvo de ações individuais, coletivas e públicas relacionadas com a prevenção e controle da obesidade abdominal em adultos no Estado de Pernambuco.

Tendo em vista a magnitude do problema da obesidade abdominal em Pernambuco e a escassez de dados representativos no país, seria relevante que pesquisas nacionais incorporassem a coleta da medida da circunferência da cintura, possibilitando, desta forma, mapear o problema nas diferentes regiões do Brasil. 


\section{Resumen}

Con el objetivo de estimar la prevalencia de obesidad abdominal y evaluar los factores asociados en adultos del estado de Pernambuco, Brasil, se realizó, en 2006, un estudio transversal, de base poblacional, involucrando a 1.580 individuos en una franja de edad de 25-59 años. La obesidad abdominal fue determinada por la circunferencia de la cintura $\geq 80 \mathrm{~cm}$ para mujeres y $\geq 94 \mathrm{~cm}$ para hombres. El modelo conceptual consideró variables socioeconómicas, demográficas, reproductivas y de comportamiento. La prevalencia de obesidad abdominal fue de un 27,1\% (IC95\%: 23,8-30,7) en el sexo masculino y un 69,9\% (IC95\%: $66,8-72,8)$ en el femenino $(p<0,001)$. El análisis ajustado reveló mayor prevalencia en hombres a partir de 50 años, de la región metropolitana, de mayor renta, ex-fumadores y consumidores de bebidas alcohólicas. Entre las mujeres, fue más prevalente a partir de 30 años, en ex-fumadoras y en aquellas con la primera gestación antes de los 18 años. Es evidente la gravedad y la multifactorialidad del problema de la adiposidad central en el estado de Pernambuco, además de ser destacable que los determinantes de la obesidad son diferentes entre los sexos.

Obesidad Abdominal; Circunferencia de la Cintura; Factores Epidemiológicos; Adulto

\section{Referências}

1. Peixoto MRG, Benicio MHD, Latorre MRDO, Jardim PCBV. Circunferência da cintura e índice de massa corporal como preditores da hipertensão arterial. Arq Bras Cardiol 2006; 87:462-70.

2. Velásquez-Melendez G, Pimenta AM, Kac G. Epidemiologia do sobrepeso e da obesidade e seus fatores determinantes em Belo Horizonte (MG), Brasil: estudo transversal de base populacional. Rev Panam Salud Pública 2004; 16:308-14.

3. Oliveira LPM, Assis AMO, Silva MCM, Santana MLP, Santos NS, Pinheiro SMC, et al. Fatores associados a excesso de peso e concentração de gordura abdominal em adultos na cidade de Salvador, Bahia, Brasil. Cad Saúde Pública 2009; 25:570-82.

4. Olinto MTA, Nácul LC, Dias-da-Costa JS, Gigante DP, Menezes AMB, Macedo S. Níveis de intervenção para obesidade abdominal: prevalência e fatores associados. Cad Saúde Pública 2006; 22:1207-15.

5. Olinto MTA, Dias-da-Costa JS, Kac G, Pattussi MP. Epidemiologia da obesidade abdominal em mulheres adultas residentes no Sul do Brasil. Arch Latinoam Nutr 2007; 57:349-56.

\section{Colaboradores}

C. P. S. Pinho contribuiu na concepção do estudo, análise e interpretação dos dados, redação do artigo. A. S. Diniz colaborou na análise, interpretação dos dados e revisão crítica do conteúdo intelectual do artigo. I. K. G. Arruda participou da análise, interpretação dos dados e redação do artigo. M. Batista Filho e P. I. C. Lira coordenaram o trabalho de campo e realizaram a revisão crítica relevante do conteúdo intelectual. P. C. Coelho e L. A. S. Sequeira colaboraram na revisão crítica relevante do conteúdo intelectual.
6. Vasques ACJ, Priore SE, Rosado LEFPL, Franceschini SCC. Utilização de medidas antropométricas para avaliação do acúmulo de gordura visceral. Rev Nutr 2010; 23:107-18.

7. Linhares RS, Horta BL, Gigante DP, Dias-da-Costa JS, Olinto MTA. Distribuição de obesidade geral e abdominal em adultos de uma cidade no Sul do Brasil. Cad Saúde Pública 2012; 28:438-46.

8. World Health Organization. Obesity: preventing and managing the global epidemic. Geneva: World Health Organization; 1998. (Report of a WHO Consultation on Obesity).

9. Barros MV, Nahas MV. Comportamentos de risco, auto-avaliação no nível de saúde e percepção de estresse entre trabalhadores da indústria. Rev Saúde Pública 2001; 35:554-63.

10. Fornés NS, Martins IS, Velásquez-Melendez G, Latorre MRDO. Escores de consumo alimentar e níveis lipêmicos em população de São Paulo, Brasil. Rev Saúde Pública 2002; 36:12-8.

11. Veloso HJF, Silva AUM. Prevalência e fatores associados à obesidade abdominal e ao excesso de peso em adultos maranhenses. Rev Bras Epidemiol 2010; 13:400-12. 
12. Pinho CPS, Diniz AS, Arruda IKG, Lira PIC, Sequeira LAS, Gonçalves FCLSP, et al. Excesso de peso em adultos do Estado de Pernambuco, Brasil: magnitude e fatores associados. Cad Saúde Pública 2011; 27:2340-50.

13. Pimenta AM, Kac G, Gazzinelli A, Corrêa-Oliveira $R$, Velásquez-Meléndez G. Associação entre obesidade central, triglicerídeos e hipertensão arterial em uma área rural do Brasil. Arq Bras Cardiol 2008; 90:419-25.

14. Ronsoni RM, Coutinho MSSA, Pereira MR, Silva RH, Becker IC, Sehnen Jr. L. Prevalência de obesidade e seus fatores associados na população de Tubarão - SC. ACM Arq Catarin Med 2005; 34:51-7.

15. World Health Organization. WHO Expert Committee on Physical Status: the use and interpretation of antropometry physical status: the use and interpretation of anthropometry. Geneva: World Health Organization; 1995.

16. Teichmann L, Olinto MTA, Dias-da-Costa JS, Ziegler D. Fatores associados ao sobrepeso e à obesidade em mulheres de São Leopoldo, RS. Rev Bras Epidemiol 2006; 19:360-73.

17. Gutierrez-Fisac JL, Regidor E, Banegas JRB, Artalejo FR. The size of obesity differences associated with educational level in Spain, 1987-1995/97. J Epidemiol Community Health 2002; 56:457-60.

18. Monteiro CA, Conde WL, Lu B, Popkin BM. Obesity and inequities in health in the developing world. Int J Obes Relat Metab Disord 2004; 28:1181-6.

19. Oliveira EO, Velásquez-Melendez G, Kac G. Fatores demográficos e comportamentais associados à obesidade abdominal em usuárias de centro de saúde de Belo Horizonte, Minas Gerais, Brasil. Rev Nutr 2007; 20:361-9.
20. Kac G, Velásquez-Meléndez G, Valente J. Menarca, gravidez precoce e obesidade em mulheres brasileiras selecionadas em um centro de saúde de Belo Horizonte, Minas Gerais, Brasil. Cad Saúde Pública 2003; 19 Suppl 1:S111-8.

21. Klein LC, Corwin EJ, Ceballos RM. Leptin, hunger, and body weight: influence of gender, tobacco smoking, and smoking abstinence. Addict Behav 2004; 29:921-7.

22. Flegal KM, Troiano RP, Pamuk ER, Kuczmarski RJ, Campbell SM. The influence of smoking cessation on the prevalence of overweight in the United States. N Engl J Med 1995; 333:1165-70.

23. Filozof C, Fernandez Pinilla MC, Fernandez-Cruz A. Smoking cessation and weight gain. Obes Rev 2004; 5:95-103.

24. Chatkin R, Chatkin JM. Tabagismo e variação ponderal: a fisiopatologia e genética podem explicar esta associação? J Bras Pneumol 2007; 33:712-9.

25. Malta DC, Moura EC, Silva SA, Oliveira PPV, Silva VLC. Prevalência do tabagismo em adultos residentes nas capitais do estado e do Distrito Federal, Brasil, 2008. J Bras Pneumol 2010; 36:75-83.

26. Oliveira Filho A, Shiromoto RN. Efeitos do exercício físico regular sobre índices preditores de gordura corporal: índice de massa corporal, relação cintura quadril e dobras cutâneas. Rev Educ Fís 2001; 12:105-15.

Recebido em 01/Fev/2012

Versão final reapresentada em 28/Ago/2012

Aprovado em 03/Out/2012 